\title{
Nonlinear Model of Coupled Superconducting Lines
}

\author{
Jordi Mateu, Carlos Collado, and Juan M. O’Callaghan
}

\begin{abstract}
High Temperature Superconductor materials are known to produce intermodulation and other nonlinear effects. In planar structures, these nonlinearities depend on the field configuration on the strip, which in turn strongly depends on the device structure and therefore on the coupling between the strips. In this work we present an equivalent circuit that models the linear and nonlinear effects in coupled transmission lines. Numerical techniques based on Harmonic Balance are used to solve the equivalent circuit. We validate the equivalent circuit model by fitting measurements of a superconducting hairpin notch resonator. Examples of several coupled transmission line structures are given.
\end{abstract}

Index Terms-Coupled lines, nonlinearities, resonator, superconductor.

\section{INTRODUCTION}

$\mathbf{T}$ HE very low surface resistance of high-temperature-superconductors (HTS) at microwave frequencies allows the fabrication of high performance miniaturized planar resonators and filters. These devices often result in novel folded topologies, which lead to significant coupling between lines.

The combination of low loss and low volume results in high current densities, which may give rise to performance degradation of resonators or filters because of the microwave nonlinearities of HTS materials. These nonlinearities are distributed along the circuit pattern and are strongly dependent on the field distribution.

The couplings among the lines of the resonators or between different resonators may have an important contribution to the current density distribution affecting the small signal performance and the nonlinear performance of the device [1].

A significant finding of [1] is the fact that, in resonators with antisymmetric (or antiparallel) currents between lines, the magnetic fields add up in the area between lines to screen the field from the superconductor. This increases the losses and the nonlinear effects in the resonator. In contrast, when the currents flow symmetrically, the fields are partially cancelled in the gap and the field configuration tends to smooth the current peaks at the edges of the lines. This has beneficial effects on the RF losses and on the nonlinearities. Therefore the nonlinearities in HTS planar resonators can be significantly reduced by designing structures which avoid strong edge currents.

Manuscript received October 4, 2004. This work was supported by the Spanish Ministry of Science and Technology through Project MAT2002-04551-C03-03, by Generalitat de Catalunya (DURSI) through Grant 2001 SGR 00266 and NEWCOM Project IST-2002-507525.

J. Mateu is with the Centre Tecnològic de Telecomunicacions de Catalunya (CTTC), Barcelona 08034, Spain (e-mail: jordi.mateu@cttc.es).

C. Collado and J. M. O'Callaghan are with Universitat Politècnica de Catalunya (UPC), Barcelona 08034, Spain (e-mail: collado@tsc.upc.es; joano@tsc.upc.es).

Digital Object Identifier 10.1109/TASC.2005.850150
In this work we have developed nonlinear circuit models for multiple coupled superconducting transmission lines. The aim of this circuit model is to be able to predict and design highperformance HTS resonators with high quality factor $\left(\mathrm{Q}_{0}\right)$ and reduced intermodulation products (IMD). Numerical techniques based on Harmonic Balance (HB) algorithms have been used to assess the nonlinear performance of the devices [2]. To validate the equivalent circuit, a superconducting hairpin notch resonator having three coupled lines (resonator arms and through line) has been examined. This structure has been studied and measured in [3].

Finally we illustrate the possibilities of the circuit model by calculating the dependence of the $\mathrm{Q}_{0}$ and IMD on the size of the gap between the lines of several folded resonant structures and also as a function of the resonant operating mode.

\section{CiRCUIT MODEL}

The nonlinear effects in an HTS transmission line can be modeled by using a distributed RLCG elemental cell model in which the resistance and inductance per unit length at a point along the line are dependent on the total current at that point [4]:

$$
L(i)=L_{0}+\Delta L(i) ; \quad R(i)=R_{0}+\Delta R(i)
$$

To evaluate the nonlinear terms $\Delta R(i)$ and $\Delta L(i)$, one needs to know the current density distribution over the cross section of the lines and the properties of the material.

The response of a single HTS transmission line can be analyzed by forming a circuit containing a cascade of many elemental cells [2].

However, as mentioned above, most miniature planar resonators consist of folded transmission lines where the coupling between lines may affect the linear and nonlinear performance. Therefore, a more elaborate approach has to be considered to address the couplings among the different transmission lines. As an example, Fig. 1 outlines a schematic structure of a meander transmission line with $\mathrm{N}$ turns.

As done for a single transmission line, the new circuit model is also based on splitting the whole circuit in many segments to consider the distributed effects (Fig. 1(b)), each of them modeling the coupling among the transmission lines.

The resulting lumped network representing multiconductor coupled lines may be derived from the matrices $\left[\mathrm{L}_{0}\right],[\mathrm{C}],\left[\mathrm{R}_{0}\right]$ and [G] [5]. The coupling between the strips due to the electric fringing fields is represented by the capacitance matrix, whereas the magnetic coupling is represented by the inductance matrix. The equivalent circuit of a segment of an $\mathrm{N}$ coupled superconducting transmission line set is outlined in Fig. 2.

The distributed parameters which characterize the linear part $\left(L_{i j}, R_{i j}, G_{i j}, C_{i j}\right.$ ) only depend on the layout, the material 


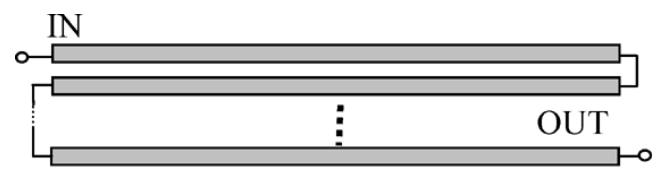

a)

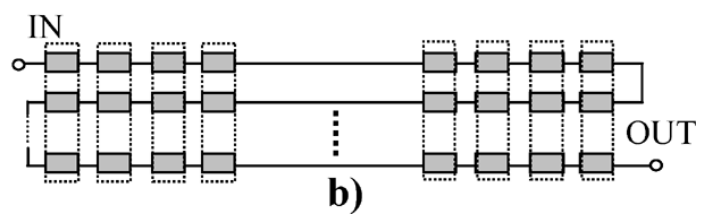

Fig. 1. (a) Schematic of meander transmission lines with $\mathrm{N}$ turns. (b) The whole device can be modeled as a concatenation of many elemental cells, each of them modeling the coupling among the transmission lines in a short longitudinal segment.

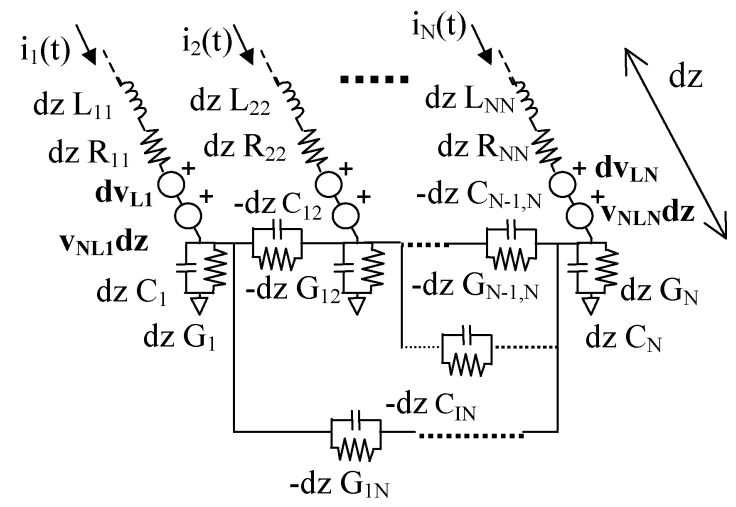

Fig. 2. Equivalent circuit of a segment of an $\mathrm{N}$ coupled transmission line set. Linear cross-coupling is modeled by voltage sources $d v_{L 1} \ldots d v_{L N}$, whereas $v_{N L 1} d z \ldots v_{N L N} d z$ model nonlinear cross-coupling effects.

properties and the frequency, so they do not depend on the voltage or on the current flowing through the lines. Thus, $L_{i j}$, $R_{i j}, G_{i j}$ and $C_{i j}$ can be used to fit several resonant modes, having different field configuration. Note that in Fig. 2, the mutual inductance and resistance between lines are taken into account by voltage sources, $d v_{L i}=\sum_{\substack{j=1 \\ j \neq i}}^{N}\left(R_{i j} i_{j}+d\left(L_{i j} i_{j}\right) / d t\right)$ [5].

As we pointed out above, the nonlinearities strongly depend on the volume current density, hence affecting the distributed parameters which models the metallic part of equivalent circuit, i.e., the resistance and inductance matrix. So that, similarly as done for a single line, we can write the overall resistance and inductance matrix per unit of length $[\mathrm{R}]$ and $[\mathrm{L}]$ as:

$$
[L]=\left[L_{0}\right]+[\Delta L([i])] ; \quad[R]=\left[R_{0}\right]+[\Delta R([i])]
$$

Where the nonlinear terms $[\Delta L]$ and $[\Delta R]$, are matrices which depend on the current flowing through the lines of the coupled structures. These currents are represented by the current vector $i$.

By using (2), we model the nonlinear contribution as a voltage $\left(v_{N L i}\right)$ source which depends nonlinearly on the current at each line of the circuit model of Fig. 2, i.e. this source takes into account the coupling between lines and the field configuration. The resulting expression of the nonlinear sources is:

$$
v_{N L_{i}}=\sum_{j=1}^{N}\left(\Delta R_{i j}\left(i_{j}\right) i_{j}+\frac{d\left(\Delta L_{i j}\left(i_{j}\right) i_{j}\right)}{d t}\right)
$$

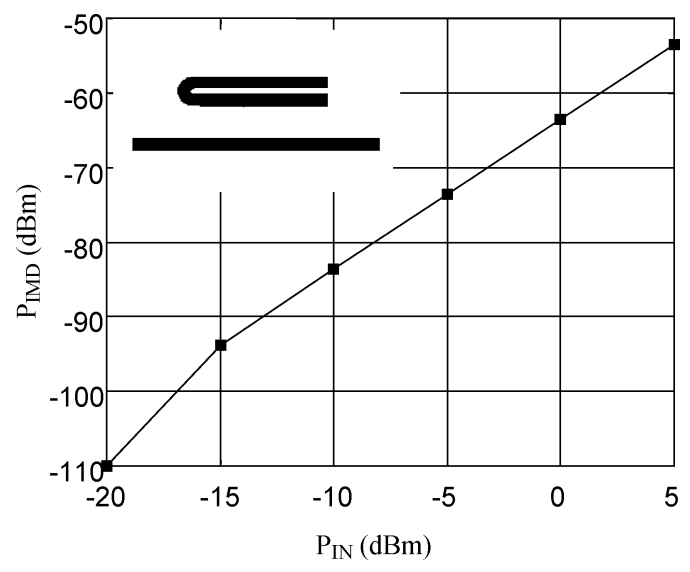

Fig. 3. Intermodulation products as a function of input power. Dots: measured data. Lines: data fitting with the models in this work. Inset: hairpin resonator layout.

Where $\Delta L_{i j}, \Delta R_{i j}$ are the components of $[\Delta L]$ and $[\Delta R]$, respectively, and $i_{j}$ the current flowing for the $j^{\text {th }}$ line. Unlike the linear parameters, $\Delta L_{i j}, \Delta R_{i j}$ depend on the field configuration in the cross section. Hence, to evaluate these, one needs to know the volume current density distribution over the cross sections of the lines. The problem is somewhat complicated and we use a numerical procedure like the one described in [4] based on [6] (we call this method WSD). Contrary to what happens with $L_{i j}, R_{i j}, G_{i j}$ and $C_{i j}$, the values of $\Delta L_{i j}, \Delta R_{i j}$ can vary from one resonant mode to another.

To simulate a whole circuit, we concatenate many elemental cells like the one in Fig. 2, and extract the corresponding nodal matrix, which allows us to obtain the impedance matrix to which we apply the HB algorithm [2].

\section{CROSS-CHECK: HAIRPIN RESONATOR}

To test the nonlinear circuit model described in the previous section, we have examined a superconducting hairpin resonator, having three coupled lines (two resonator arms coupled to a through line), as shown in inset of Fig. 3. Besides being a simple test device, superconducting hairpin resonators have been thoroughly studied and measured in [3], and their intermodulation properties are well characterized.

Fig. 3 reproduces data in Fig. 3 of [3] (circles) and the fitting (solid line) done using the model of Fig. 2. For power levels up to $-15 \mathrm{dBm}$, the data have been fit with a square-law dependence of the nonlinear parameters on the current flowing through the line [4], that is: $\Delta L_{i j}\left(i_{j}\right)=\Delta L_{i j} i_{j}^{2} ; \Delta R_{i j}\left(i_{j}\right)=\Delta R_{i j} i_{j}^{2}$.

Whereas at higher power levels, modulo nonlinearities [2] have been used, i.e., $\Delta L_{i j}\left(i_{j}\right)=\Delta L_{i j}\left|i_{j}\right|$; $\Delta R_{i j}\left(i_{j}\right)=\Delta R_{i j}\left|i_{j}\right|$.

We have assumed that the nonlinearities come from the dependence of the penetration depth on the volume current density [4], as $\lambda_{L}^{2}(T, j)=\lambda_{L}^{2}(T, 0)(1-f(T, j))^{-1}$, where $f(T, j)=$ $\left(j / j_{I M D}\right)^{2}$ for a quadratic dependence and $f(T, j)=$ $\left|j / j_{I M D}^{\prime}\right|$ for a modulus dependence. The values which fit the measurements of Fig. 3 are $j_{I M D}=1.5 \cdot 10^{11} \mathrm{~A} \cdot \mathrm{m}^{-2}$ and $j_{I M D}^{\prime}=5 \cdot 10^{12} \mathrm{~A} \cdot \mathrm{m}^{-2}$, respectively. The nonlinear circuital parameters, $\Delta L_{i j}, \Delta R_{i j}$, obtained from these values, using WSD, demonstrate the current flowing along the through line does not affect the nonlinearites of the hairpin resonator. 


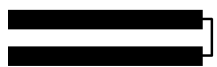

a)

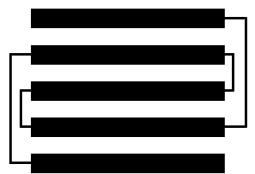

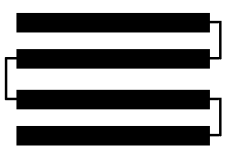

b)

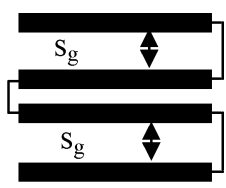

c) d)

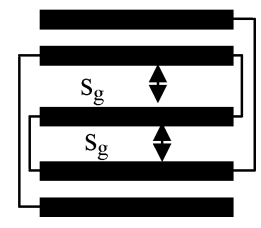

e)

Fig. 4. Geometry of different resonators. a) Hairpin line. b) 4th order meander line. c) Special 4th order meander line. d) 5th order spiral-in-spiral-out [1]. e) special 5th order spiral-in-spiral-out.

These measurements have also been fitted by using closedform expression [2], validating the equivalent nonlinear circuit model.

\section{Examples With More Complex Structures}

To illustrate some possibilities of the study developed above, in this section we consider various folded resonant structures, see Fig. 4. We examine the dependence of the $\mathrm{Q}_{0}$ and IMD on the size of the gap between the coupled lines $(g)$ of these structures. We also compare the differences between the performance of the devices when they are excited either with the first (1 HM) or the second harmonic mode (2 HM). These are the resonant frequencies corresponding to a half-wave length and a wave length, respectively.

The material properties taken for the analysis are: $700 \mathrm{~nm}$ thickness YBCO, deposited on both side of a $500 \mu \mathrm{m} \mathrm{MgO}$ substrate. Surface resistance and loss tangent are $\mathrm{R}_{\mathrm{s}}(77 \mathrm{~K})=$ $15 \mu \Omega$ at $2 \mathrm{GHz}$ and $\tan \delta=5 \cdot 10^{-6}$. The length of the arms of the resonators are adjusted to resonate at $2 \mathrm{GHz}$ either for the 1 $\mathrm{HM}$ or the $2 \mathrm{HM}$. The width of the lines is fixed at $w=500 \mu \mathrm{m}$.

The distributed parameters $\left(L_{i j}, R_{i j}, C_{i j}, G_{i j}, \Delta L_{i j}, \Delta R_{i j}\right.$, see Fig. 2), to model the layouts have been obtained using the WSD method where we consider a square-law dependence of the penetration depth on the current density with a scaling parameter, $\mathrm{j}_{\mathrm{IMD}}=1.5 \times 10^{11} \mathrm{~A} / \mathrm{m}^{2}$ [2].

We have also considered that the input and output coupling coefficients are identical in all the structures, thus for a given input power, the power dissipated in the resonator and the power coupled out of it, at the fundamental frequencies will be the same in all cases. However, the loaded quality factors of the studied structures will be different and we should keep in mind that the IMD coupled out of the cavity will take into account, both, the nonlinear effects $\left(\Delta \mathrm{L}_{\mathrm{ij}}\right.$ and/or $\left.\Delta \mathrm{R}_{\mathrm{ij}}\right)$ and the losses $\left(\mathrm{Q}_{0}\right)[1]$.

The results of the sets of simulations are depicted in Figs. 5, 7 and 8, all of them compare the $\mathrm{Q}_{0}$ (left side) and IMD (right side) as a function of $g$.

Fig. 5 shows the results for the structures of Fig. 4(a), circles and Fig. 4(b), squares, for the $1 \mathrm{HM}$, unfilled marks, and for the $2 \mathrm{HM}$, filled marks. From these results we see that:

- Operating at the $1 \mathrm{HM}$ : The values of $\mathrm{Q}_{0}$ and IMD of the hairpin and the 4th order meander line resonator are similar. This is because the most important contribution of
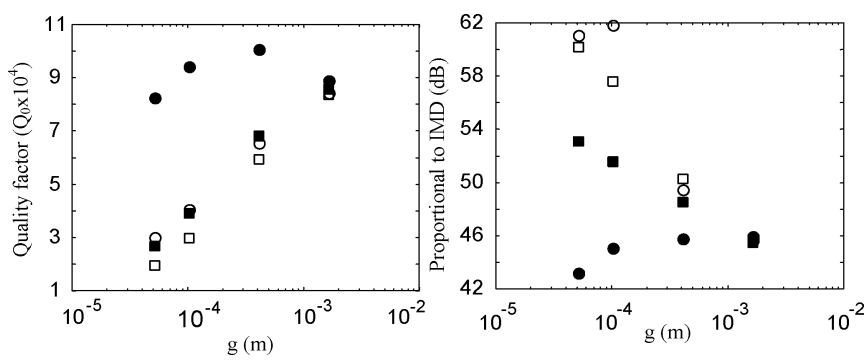

Fig. 5. $\quad \mathrm{Q}_{0}$ (left side) and IMD (right side) as a function of the gap for the structures of Fig. 4(a), circles and Fig. 4(b), squares. Unfilled marks indicate first harmonic mode and filled marks indicate second harmonic mode.

a)

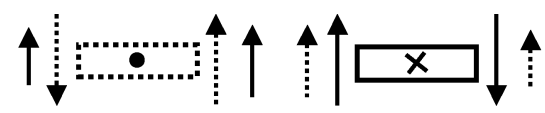

)

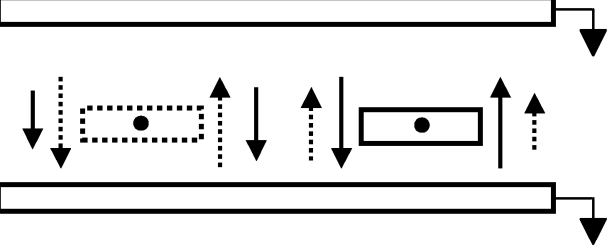

Fig. 6. Magnetic field on the edges of the strips of a two coupled lines in a microstrip structure. a) Currents flowing antisymmetrically, b) currents flowing symmetrically.

the nonlinear effects takes place where the current density is largest, which in the $1 \mathrm{HM}$ is in the middle of the line.

- Operating at the $2 \mathrm{HM}$ : When the inter-line spacing is reduced: a) The $\mathrm{Q}_{0}$ of the hairpin resonator degrades little in comparison with the 4 th order meander line. Note that when $g$ increases the current distribution tends to be like the one in a isolated line of $w$, which shows a sharper profile than the one observed in coupled lines with symmetrical currents [3]. b) The IMD of the hairpin resonator improves, instead of the observed in the 4th order meander line. This can be explained from the symmetric currents running along two arms of the hairpin [1], [5]. For a better understanding, Fig. 6 outlines the magnetic field on the edges of a two coupled lines, representing the cross section of a hairpin structure. Fig. 6(a) corresponds to the $1 \mathrm{HM}$, when the currents flow antisymmetrically. In this case the magnetic field adds up at the areas between lines. Whereas for the $2 \mathrm{HM}$, when the currents flow in symmetrically, outlined in Fig. 6(b), the fields are cancelled in the gap. Note that this can be done for any of the structures of Fig. 4.

An example to confirm the effects of antisymmetric or symmetric current flowing between the arms of the structures is the one shown in Fig. 7. It shows how the performance of the resonator can be improved by simply moving away the arms which hold an antisymmetric currents. Fig. 7 depicts the $\mathrm{Q}_{0}$ and IMD of a hairpin resonator (Fig. 4(a)), circle, a 4th meander line (Fig. 4(b)), squares, and an special 4th meander line (Fig. 4(c)), diamonds, where $\mathrm{s}=1.6 \mathrm{~mm}$ (more than three times the width line). All the resonators operate at $2 \mathrm{HM}$. The resonator of Fig. 4(b) exhibits the poorest performance with highest nonlinearities and lowest $\mathrm{Q}_{0}$ (square marks). Note that this is 

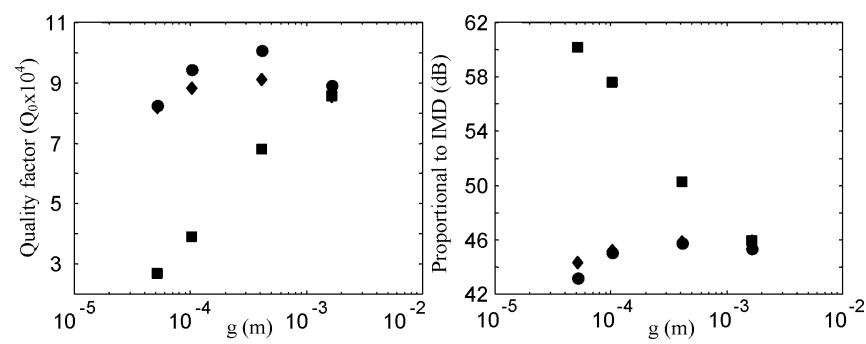

Fig. 7. $\mathrm{Q}_{0}$ (left side) and IMD (right side) as a function of the gap for the structures of Fig. 4(a), circles, Fig. 4(b), squares, and Fig. 4(c), diamonds. All cases are considered operating at second harmonic mode. Note that the gaps between arms holding antisymmetric current in the resonator of Fig. 4(c) are kept fixed at $1.6 \mathrm{~mm}$.
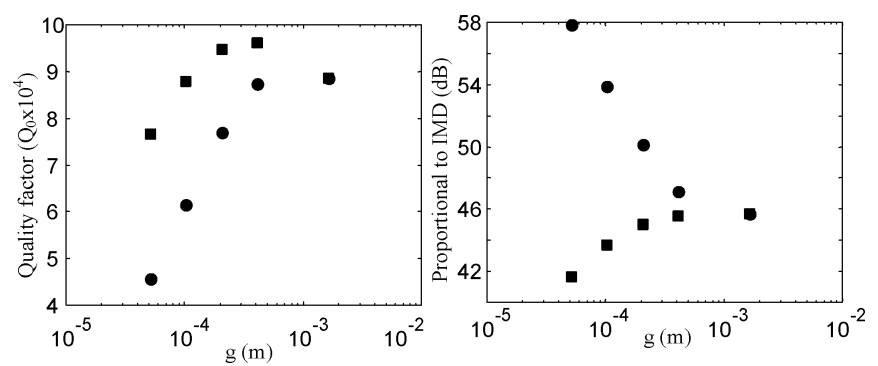

Fig. 8. $\mathrm{Q}_{0}$ (left side) and IMD (right side) as a function of the gap for the structures of Fig. 4(d), circles, Fig. 4(e), squares. Both cases are studied for the second harmonic mode. Note that the central gaps at the resonator of Fig. 4(e) are kept fixed at $1.6 \mathrm{~mm}$ and only the other gaps are changed.

because the current runs antisimmetrically between close arms of the resonator of Fig. 4(b).

The example of Fig. 8, as the preceding one, evaluates the effects of changing the spacing between lines on $\mathrm{Q}_{0}$ and IMD in structures like the ones depicted in Fig. 4(d) and Fig. 4(e). When we keep the central line far away (more than three times the width line, $\mathrm{s}_{\mathrm{g}}=1.6 \mathrm{~mm}$ ) from the others with antisymmetric currents, then providing low IMD.

To summarize, the simulations presented in this section show that resonators with coupled lines having symmetric currents have good nonlinear properties due to smoothing effects. Besides these distributions increase the ratio between energy stored and losses, thus increase the $\mathrm{Q}_{0}$ of the structures. On the other hand, we should notice that the most important contribution of the nonlinear effects take place where the current density is largest, hence one could reduce the IMD by enlarging the width line in the areas of the structure where the peak current density is largest.

Note that these conclusions have already pointed out in the experiment outlined in [1]. This work provides an alternative confirmation, and a method to predict the performance of other resonators based on coupled transmission lines.

\section{CONCLUSION}

A nonlinear circuit model for multiple coupled transmission lines has been developed and successfully cross-checked against experimental data. This method also allows us to study the dependence of the losses and the IMD power on the geometry of the resonators, particularly the size of the gap between the coupled lines and the mode excited in the structure. After these cross-checks, the models are ready to make realistic simulations of other superconducting devices with coupled lines.

\section{ACKNOWLEDGMENT}

The authors acknowledge Superconducting Technologies Inc. for hosting J. Mateu during his Ph.D work.

\section{REFERENCES}

[1] B. Willemsen, A. Cardona, N. Fenzi, and R. Forse, "High Temperature Superconducting Structures and Methods for High Q, Reduced Intermodulation Resonator and Filters," U.S. Patent 6026311 , Feb. 15, 2000

[2] C. Collado, J. Mateu, and J. M. O'Callaghan, "Analysis and simulation of the effects of distributed nonlinearities in microwave superconducting devices," IEEE Trans. Appl. Superconduct., to be published.

[3] B. A. Willemsen, T. Dahm, and D. J. Scalapino, "Microwave intermodulation thin film high-Tc superconducting microstrip hairpin resonators: Experiment and theory," Appl. Phys. Lett., vol. 71, no. 26, pp. 3898-3900, 1997.

[4] T. Dahm and D. J. Scalapio, "Theory of intermodulation in a superconducting microstrip resonator," J. Appl. Phys., vol. 81, pp. 2002-2009, 1997.

[5] C. Wei, R. Harrington, J. Mautz, and T. Sarkar, "Multiconductor transmission lines in multilayered dielectric media," IEEE Trans. Microw. Theory Tech., vol. MTT-32, no. 4, pp. 439-450, 1984.

[6] D. M. Sheen, S. M. Ali, D. E. Oates, R. S. Withers, and J. A. Kong, "Current distribution, resistance and inductance for superconducting strip lines," IEEE Trans. Appl. Superconduct., vol. 1, pp. 108-115, 1991. 